\title{
Ultra-high-resolution computed tomography can demonstrate alveolar collapse in novel coronavirus (COVID-19) pneumonia
}

\author{
Tae Iwasawa ${ }^{1,3} \cdot$ Midori Sato $^{2} \cdot$ Takafumi Yamaya $^{2} \cdot$ Yozo Sato $^{2} \cdot$ Yoshinori Uchida $^{2} \cdot$ Hideya Kitamura $^{2} \cdot$ Eri Hagiwara $^{2}$. \\ Shigeru Komatsu ${ }^{2} \cdot$ Daisuke Utsunomiya ${ }^{1,3} \cdot$ Takashi Ogura $^{2}$
}

Received: 6 March 2020 / Accepted: 10 March 2020 / Published online: 31 March 2020

(c) Japan Radiological Society 2020

\begin{abstract}
Purpose To review the chest computed tomography (CT) findings on the ultra-high-resolution CT (U-HRCT) in patients with the Novel coronavirus disease 2019 (COVID-19).

Materials and Methods In February 2020, six consecutive patients with COVID-19 pneumonia (median age, 69 years) underwent U-HR CT imaging. U-HR-CT has a larger matrix size of $1024 \times 1024$ thinner slice thickness of $0.25 \mathrm{~mm}$ and can demonstrate terminal bronchioles in the normal lungs; as a result, Reid's secondary lobules and their abnormalities can be identified. The distribution and hallmarks (ground-glass opacity, consolidation with or without architectural distortion, linear opacity, crazy paving) of the lung opacities on U-HRCT were visually evaluated on a $1 \mathrm{~K}$ monitor by two experienced reviewers. The CT lung volume was measured, and the ratio of the measured lung volume to the predicted total lung capacity (predTLC) based on sex, age and height was calculated.

Results All cases showed crazy paving pattern in U-HRCT. In these lesions, the secondary lobules were smaller than those in the un-affected lungs. CT lung volume decreased in two cases comparing predTLC.

Conclusion U-HRCT can evaluate not only the distribution and hallmarks of COVID-19 pneumonia but also visualize local lung volume loss.
\end{abstract}

Keywords Novel coronavirus $\cdot$ Ultra-high-resolution computed tomography $\cdot$ Crazy-paving pattern $\cdot$ Pneumonia

\section{Introduction}

Novel coronavirus disease (COVID-19) was first reported in Wuhan, China in December 2019 [1]. It spread rapidly to the neighboring countries, including Japan, and worldwide. Until March, 2020, the total number of reported cases was 90,870, with 3112 patient deaths based on the WHO situation report-43 [2]. Computed tomography (CT) findings and their subsequent changes have been reported from China; bilateral peripheral ground glass opacities (GGO),

Tae Iwasawa

tae_i_md@wb3.so-net.ne.jp

1 Department of Radiology, Kanagawa Cardiovascular and Respiratory Center, 6-16-1, Tomioka-higashi, Kanazawa-ku, Yokohama 236-0051, Japan

2 Department of Respiratory Medicine, Kanagawa Cardiovascular \& Respiratory Centerr, Yokohama, Japan

3 Departement of Diagnostic Radiology, Yokohama City University, School of Medicine, Yokohama, Japan consolidation, and linear shadows are the characteristic CT findings in COVID-19 pneumonia [3-7].

In this study, we evaluated ultra-high-resolution CT (U-HR-CT) images of COVID-19 pneumonia to analyze the imaging characteristics in detail. U-HR-CT has both a detector element and X-ray tube focus size smaller than those in conventional CT [8]. At the same time, a deep learning reconstruction (DLR) algorithm can reduce noise. Based on these new techniques, a larger matrix size of $1024 \times 1024$ and thinner slice thickness of $0.25 \mathrm{~mm}$ can be achieved within a standard radiation dose. In U-HR-CT, the terminal bronchioles in the normal lungs can be identified, especially in the subpleural region; therefore, the Reid's secondary lobules, which are approximately equal in size-about $1 \mathrm{~cm}-$ can be recognized throughout the whole lung. Based on this assumption, we believe that U-HR-CT can easily detect local alveolar collapse.

To our knowledge, this is the first report of the use of U-HR-CT in detecting alveolar collapse in COVID-19 pneumonia. 


\section{Subjects and methods}

Our institutional review board approved this retrospective single-center study and waived the requirement for obtaining informed patient consent (KCRC-19-0050). There were six continuous cases of two male and four female patients, all aged between 63 and 71 years (median age 69 years). The patient characteristics are shown in Table 1. Throat swabs of all patients tested positive for COVID-19 using real-time fluorescence polymerase chain reaction (PCR). CT was performed 5-7 days after onset of symptoms. Two patients had no symptom. Oxygen saturation (SaO2) was more than $95 \%$ during the CT examinations in all patients. In cases three and five, however, hypoxemia had progressed to $80 \%$ of $\mathrm{SaO} 2$ the following day, and oxygen therapy was started.

All CT images were obtained using U-HR-CT (Canon Medical systems, Otawara, Japan), at full inspiration and a tube voltage of $120 \mathrm{kVp}$ with automatic tube current modulation. The CT images were reconstructed with a $1024 \times 1024$ matrix size and $0.25 \mathrm{~mm}$ slice thickness with DLR algorithm. Two expert radiologists analyzed the U-HRCT images using a workstation with a $1 \mathrm{~K}$ display monitor (ZIO station 2, Ziosoft, Inc, Tokyo, Japan) that provided multi-planar reconstruction images promptly. The radiologists consensually evaluated the distribution and hallmarks (ground-glass opacity [GGO], consolidation, linear opacity, crazy paving) of the lung opacities, and the presence of local lung volume loss. The "crazy paving pattern" is characterized was defined by as the reticular interlobular septa thickening within the patchy GGO. Moreover, we measured CT lung volume and calculated its ratio to the predicted total lung capacity (predTLC), based on sex, age, and body height.

\section{Results}

All patients had multiple bilateral lesions in the lungs, such as ground glass opacities (6/6 cases), consolidation (6/6), linear opacities (3/6), and reticulation (1/6) (Table 1). The lesions showed peripheral distribution in three cases, peri bronchial distribution in one case, and peripheral and peri bronchial distributions in other two cases.

The "crazy-paving pattern" was identified in all cases using magnified multi-planar reconstruction (Figs. 1, 2, 3). We found that each polygon corresponding to the secondary lobules was smaller than $1 \mathrm{~cm}$ in these lesions. The lobules next to the

Table 1 Patient characteristics and U-HR-CT findings

\begin{tabular}{|c|c|c|c|c|c|c|}
\hline Case & No.1 & No. 2 & No.3 & No.4 & No.5 & No.6 \\
\hline Sex & $\mathrm{F}$ & $\mathrm{F}$ & $\mathrm{F}$ & M & M & $\mathrm{F}$ \\
\hline Age & 70 & 63 & 68 & 70 & 71 & 71 \\
\hline Smoking & Ex-smoker & Never & Never & Never & Never & Never \\
\hline Comorbidity & $\begin{array}{l}\text { Hypo- } \\
\text { thyroidism } \\
\text { Asthma }\end{array}$ & Dyslipidemia & $\begin{array}{l}\text { Hypo-thyroidism } \\
\text { Fybromyalgia IBS }\end{array}$ & $\begin{array}{c}\text { Dys-lipidemia } \\
\text { Skin cancer }\end{array}$ & $\begin{array}{l}\text { Diabetes Hyperten- } \\
\text { sion CHF CKD }\end{array}$ & Asthma \\
\hline Symptoms & No & No & Fever & Fever & Fever Fatigue & Fever Fatigue \\
\hline Oxygen therapy & No & No & Yes & No & Yes & No \\
\hline White cell count $(/ \mu \mathrm{l})$ & 7360 & 5110 & 4420 & 3320 & 7450 & 3080 \\
\hline Lymphocyte count $(/ \mu \mathrm{l})$ & 2340 & 1530 & 920 & 1460 & 740 & 610 \\
\hline $\mathrm{CRP}(\mathrm{mg} / \mathrm{dl})$ & 0.80 & 0.01 & 5.86 & 0.09 & 8.51 & 1.99 \\
\hline CT timing (days) & Unknown & Unknown & 6 & 7 & 5 & 5 \\
\hline \multicolumn{7}{|l|}{ CT findings } \\
\hline Distribution & Both & Peripheral & Peripheral & Both & BVB & Peripheral \\
\hline Affected lobes & 5 & 5 & 5 & 3 & 5 & 5 \\
\hline GGO & Yes & Yes & Yes & Yes & Yes & Yes \\
\hline Consolidation & Yes & Yes & Yes & Yes & Yes & No \\
\hline Linear opacities & No & Yes & No & Yes & Yes & No \\
\hline Crazy paving pattern & Yes & Yes & Yes & Yes & Yes & Yes \\
\hline CT lung volume (ml) & 3714 & 3789 & 2915 & 4826 & 4262 & 3865 \\
\hline CTLV/predTLC (\%) & 92.3 & 98.5 & 71.0 & 81.3 & 78.5 & 96.2 \\
\hline
\end{tabular}

IBS Irritable bowel syndrome, $C H F$ Chronic heart failure, $C K D$ Chronic kidney disease, $C R P$ C-reactive protein, $C T$ timing CT timing after the onset of symptoms, $G G O$ ground glass opacities, $C T L V$ CT lung volume, predTLC predicted total lung capacity; Both, Peripheral and peri bronchial distribution, Peripheral Peripheral distribution, $B V B$ Peri bronchial distribution 

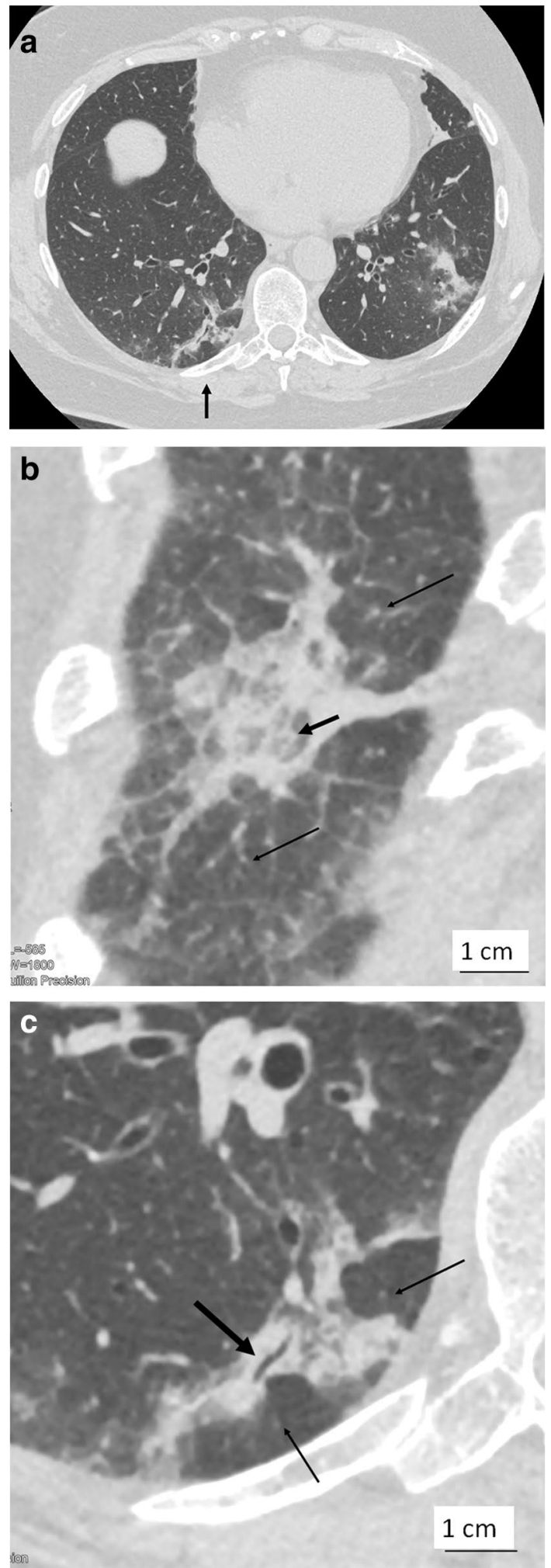

Fig. 1 U-HR-CT images of a 70-year-old female patient (Case 1). a Axial image; $\mathbf{b}$ and magnified coronal images. a The axial image shows patchy consolidation in the bilateral lower lobe. b Magnified coronal image shows terminal bronchioles which distribute Reid's secondary lobules (arrows). The size of affected lobule (thick arrow) is smaller than that of unaffected lobules (thin arrows). c Magnified axial image shows hyperinflated lobules adjacent to the consolidation (arrows)
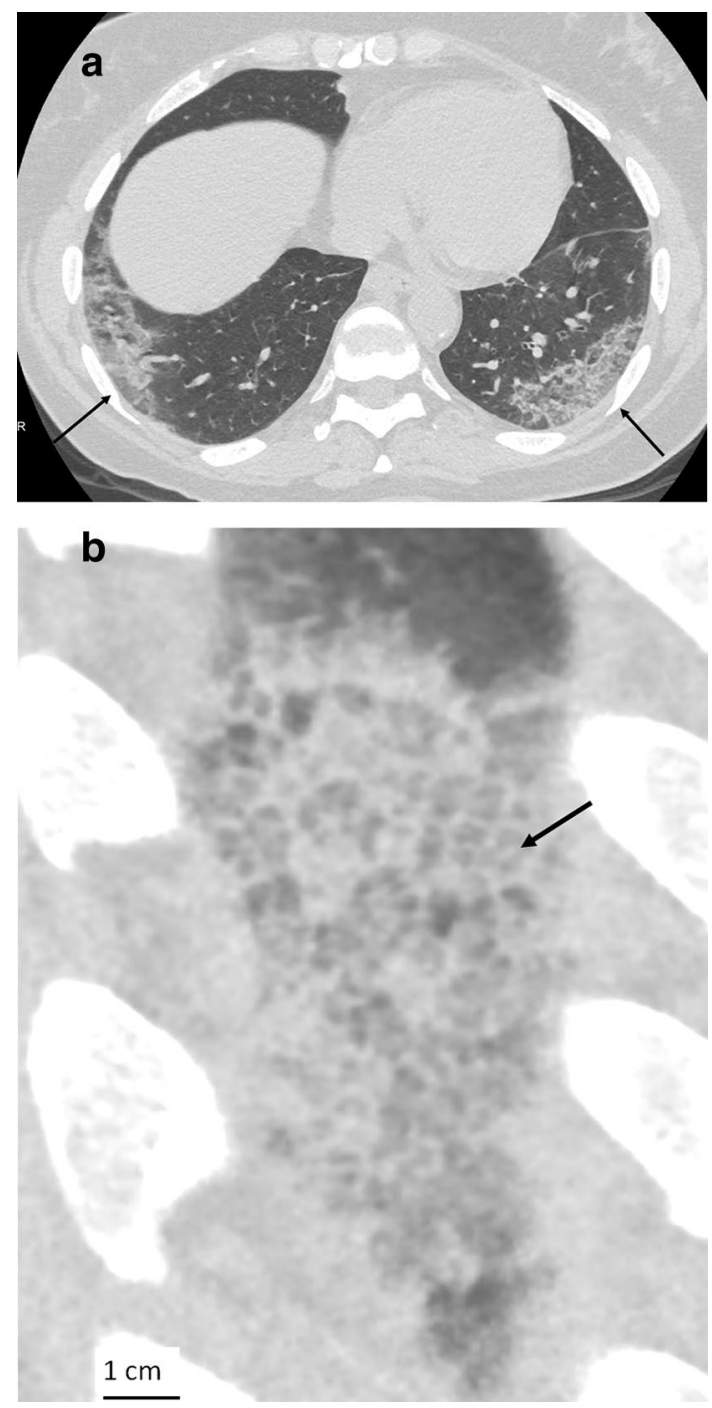

Fig. 2 U-HR-CT images of a 68-year-old female patient (Case 3). a Axial image; b, magnified coronal image. a Axial image shows bilateral peripheral ground glass opacities. b Magnified coronal image shows "crazy-paving appearance" in the ground glass opacities. One polygon (arrow) is corresponding to the Reid's secondary lobules, the diameter of polygon is smaller than $1 \mathrm{~cm}$. The CT lung volume of the patient was $71 \%$ of her predicted total lung capacity

lesion were stretched (Fig. 1). As shown in Fig. 3, the peripheral bronchi were close to the pleura, indicating that the surrounding consolidation would be accompanied with collapse.

In quantitative analysis, CT lung volume decreased to $71 \%$ in patient 3 and $78.5 \%$ in patient 5 , who needed oxygen therapy. 
Fig. 3 U-HR-CT images of a 71-year-old male patient (Case 5). a Axial image; b, coronal image; and $\mathbf{c}$, magnified sagittal images of the right middle lobe. a Axial image shows consolidation and ground glass opacities along the broncho-vascular bundles in the bilateral lungs (arrows). Dilated peripheral bronchi are found near the pleura (white arrow), which suggests consolidation to be accompanied with collapse. b Coronal image shows linear opacity in the right lower lobe. c Magnified sagittal image shows "crazy-paving appearance" which pulls the interlobar pleura. In an unaffected area, terminal bronchioles are apart from the pleura about $5 \mathrm{~mm}$ (thin arrow). In the lesions, terminal bronchioles are close to the pleura (thick arrow) and polygons of Reid's secondary lobules are smaller than $1 \mathrm{~cm}$ in diameter

\section{Discussion}

We report the U-HR-CT findings of COVID-19 pneumonia. The observed CT abnormalities were GGO, consolidation, linear opacities, and crazy-paving pattern, and lesions distributed in peripheral lung and/or peri-bronchial regions. These findings were compatible with those of the previous studies [3-7].

We observed smaller CT lung volume in severe cases. Furthermore, U-HR-CT showed that secondary lobes in the crazy-paving pattern were smaller than in unaffected lungs. These results indicated that the lesions of COVID19 pneumonia are accompanied with local volume loss. Wu et al. [7] reported that these lesions frequently pulled the adjacent pleura. Allbarello et al. reported decreased normal lung volume in COVID-19 pneumonia patients with acute respiratory distress syndrome (ARDS) [9]. We believe this volume loss resulted due to alveolar collapse, which is commonly seen in ARDS [10].

Some infections, like influenza $\mathrm{A}(\mathrm{H} 1 \mathrm{~N} 1)$ infection for example, may induce ARDS [11], such as Severe acute respiratory syndrome (SARS) [12] and COVID-19 [13]. Guan et al. [14] reported that among the 1099 patients with COVID-19, 5.0\% were admitted to the ICU, $2.3 \%$ underwent invasive mechanical ventilation, and $1.4 \%$ died. In COVID-19, virus particles spread through the respiratory mucosa and infect other cells, induce a cytokine storm in the body, generate a series of immune responses, and cause changes in peripheral white blood cells and immune cells, such as lymphocytes [13]. A few patients progress rapidly to ARDS [15]. Many clinicians seek the factors associated with rapid progression.

The lung volume measured by CT is well correlated with pulmonary function test results such as total lung capacities and forced vital capacities [16]. Decreased CT lung volume might be an important finding indicating disease severity. Thus, we believe that U-HR-CT would be useful in the detection of the abnormalities accompanied with local lung volume loss, as early identification and timely treatment of critical cases is of crucial importance.
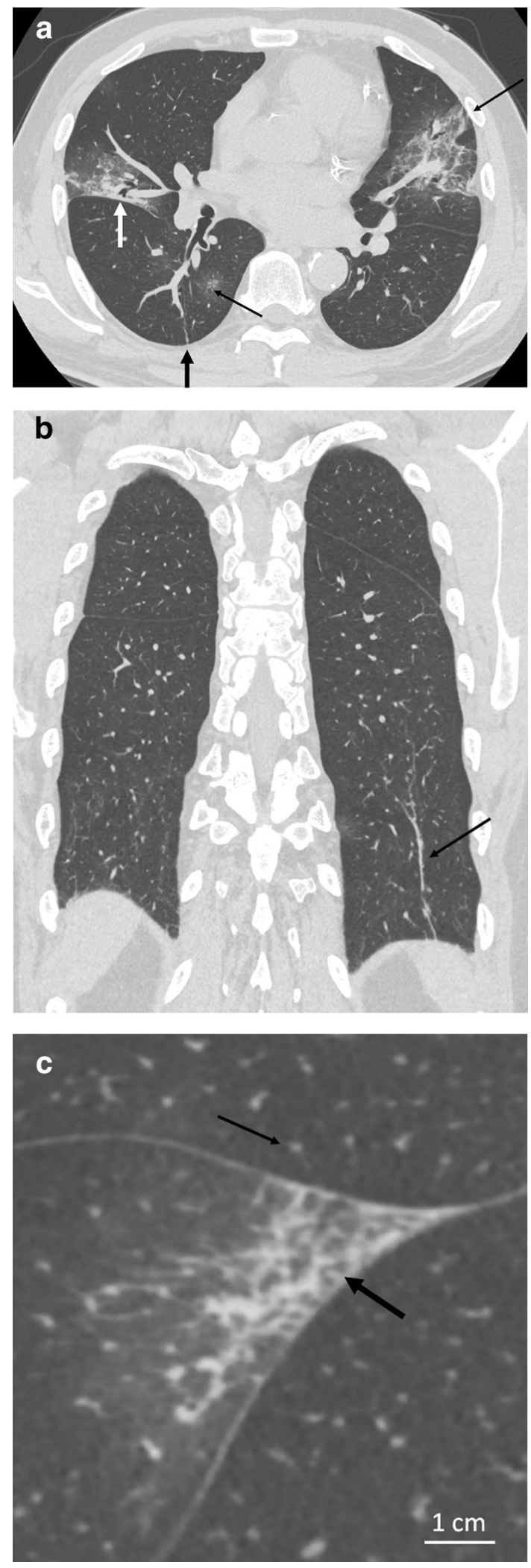

This is a short report based on limited patients in a single center. Further studies will be required to confirm the findings. 


\section{Compliance with ethical standards}

Conflict of interest We have no conflict of interest about this work.

Ethical statement All procedures performed in studies involving human participants were in accordance with the ethical standards of the institutional and/or national research committee and with the 1964 Helsinki declaration and its later amendments or comparable ethical standards.

\section{References}

1. Huang C, Wang Y, Li X, Ren L, Zhao J, Hu Y, et al. Clinical features of patients infected with 2019 novel coronavirus in Wuhan. China Lancet. 2020;395:497-506. https://doi.org/10.1016/s0140 $-6736(20) 30183-5$.

2. Coronavirus disease 2019 (COVID-19) Situation Report-37 Data as reported by $10 \mathrm{AM}$ CET 3 March 2020. https://www.who.int/ docs/default-source/coronaviruse/situation-reports/20200303-sitre p-43-covid-19.pdf?sfvrsn=2c21c09c_2. Accessed 3 Mar 2020.

3. Lei J, Li J, Li X, Qi X. CT imaging of the 2019 novel coronavirus (2019-nCoV) pneumonia. Radiology. 2020;295(1):18. https://doi. org/10.1148/radiol.2020200236.

4. Chung M, Bernheim A, Mei X, Zhang N, Huang M, Zeng X, et al. CT imaging features of 2019 novel coronavirus (2019-nCoV). Radiology. 2020;295(1):202-7. https://doi.org/10.1148/radio 1.2020200230 .

5. Pan F, Ye T, Sun P, Gui S, Liang B, Li L, et al. Time course of lung changes on chest CT during recovery from 2019 novel coronavirus (COVID-19) pneumonia. Radiology. 2020; https:// doi.org/10.1148/radiol.2020200370.

6. Wang D, Hu B, Hu C, Zhu F, Liu X, Zhang J, et al. Clinical characteristics of 138 hospitalized patients with 2019 novel coronavirus-infected pneumonia in Wuhan China. JAMA. 2020. https ://doi.org/10.1001/jama.2020.1585.

7. Wu J, Wu X, Zeng W, Guo D, Fang Z, Chen L, et al. Chest CT findings in patients with corona virus disease 2019 and its relationship with clinical features. Invest Radiol. 2020. https://doi. org/10.1097/RLI.0000000000000670.

8. Yanagawa M, Hata A, Honda O, Kikuchi N, Miyata T, Uranish A, et al. Subjective and objective comparisons of image quality between ultra-high-resolution CT and conventional area detector $\mathrm{CT}$ in phantoms and cadaveric human lungs. Eur Radiol. 2018;28:5060-8. https://doi.org/10.1007/s00330-018-5491-2.

9. Albarello F, Pianura E, Di Stefano F, Cristofaro M, Petrone A, Marchioni L, et al. 2019-novel coronavirus severe adult respiratory distress syndrome in two cases in Italy: an uncommon radiological presentation. Int J Infect Dis. 2020. https://doi. org/10.1016/j.ijid.2020.02.043.

10. Sahetya SK, Goligher EC, Brower RG. Fifty Years of research in ARDS. Setting positive end-expiratory pressure in acute respiratory distress syndrome. Am J Respir Crit Care Med. 2017;195(11):1429-38. https://doi.org/10.1164/rccm.20161 0-2035CI.

11. Kumar A, Zarychanski R, Pinto R, Cook DJ, Marshall J, Lacroix J, et al. Critically ill patients with 2009 influenza A(H1N1) infection in Canada. JAMA. 2009;302(17):1872-9. https://doi.org/10.1001/ jama.2009.1496.

12. Luyt CE, Combes A, Trouillet JL, Nieszkowska A, Chastre J. Virus-induced acute respiratory distress syndrome: epidemiology, management and outcome. Presse Med. 2011;40:e561-e568568. https://doi.org/10.1016/j.lpm.2011.05.027.

13. Xu Z, Shi L, Wang Y, Zhang J, Huang L, Zhang C, et al. Pathological findings of COVID-19 associated with acute respiratory distress syndrome. Lancet Respir Med. 2020. https://doi.org/10.1016/ S2213-2600(20)30076-X.

14. Guan W, Ni Z, Hu Y, Liang W, Ou C, He J, et al. Clinical characteristics of coronavirus disease 2019 in China. N Engl J Med. 2020. https://doi.org/10.1056/NEJMoa2002032.

15. Chen N, Zhou M, Dong X, Qu J, Gong F, Han Y, et al. Epidemiological and clinical characteristics of 99 cases of 2019 novel coronavirus pneumonia in Wuhan, China: a descriptive study. Lancet. 2020;395:507-13. https://doi.org/10.1016/s0140-6736(20)30211 $-7$.

16. Robbie H, Wells AU, Jacob J, Walsh SLF, Nair A, Srikanthan A, et al. Visual and automated CT measurements of lung volume loss in idiopathic pulmonary fibrosis. AJR Am J Roentgenol. 2019;213(2):318-24. https://doi.org/10.2214/AJR.18.20884.

Publisher's Note Springer Nature remains neutral with regard to jurisdictional claims in published maps and institutional affiliations. 